\title{
Chemical ecology of insect parasitoids: towards a new era
}

\section{Stefano Colazza ${ }^{1}$ and Eric Wajnberg ${ }^{2}$}

${ }^{1}$ Department of Agricultural and Forest Sciences, University of Palermo, Italy ${ }^{2}$ INRA, Sophia Antipolis Cedex, France

\begin{abstract}
Over the course of evolutionary time, insect parasitoids have developed diverse strategies for using chemical compounds to communicate with various protagonists within their environment (i.e. conspecifics, their hosts, and the plants on which their hosts are livingl. Unravelling the evolutionary meaning of such chemical communication networks not only provides new insights into the ecology of these insects but also contributes to improving the use of parasitoids for the control of insect pests in biological control programmes. A book covering our current knowledge of the chemical ecology of insect parasitoids is therefore particularly timely and will appeal to a large number of potential readers worldwide, from university students to senior scientists. Internationally recognized specialists were invited to contribute chapters to this book, examining the main topics and exploring the most interesting issues in the field of chemical ecology of insect parasitoids. The chapters are organized so as to present the most significant knowledge and discoveries made over recent decades, and their potential uses in pest control.
\end{abstract}

\subsection{Introduction}

For several million years, plants, insects and their natural enemies have coevolved on the basis of information flows within food webs (Krebs \& Davies 1987). As a consequence, they were - and still are - continuously exposed to selection pressures which drive evolution according to a process referred to as an 'arms race' (Dawkins \& Krebs 1979). Different ecological features of interacting species can evolve in response to selection pressures, leading species and their populations to evolve and improve their reproductive success. 
Most of the time, such responses cause individuals to react more effectively to signals coming from their biotic and abiotic environment. Among the different types of signals that can influence these ecological interactions, chemical cues, called semiochemicals (from the Greek 'semeion', a mark or signal), play the major role (Nordlund 1981, Vinson 1985, Vet \& Dicke 1992). These compounds can be classified into two groups, named pheromones and allelochemicals.

Pheromones (from the Greek 'pherein', to carry, and 'horman', to excite or stimulate) are chemical signals that mediate interactions between individuals of the same species, and they are often described on the basis of their function (Wyatt 2010). Since their discovery, many pheromones have been identified and synthesized and a number of techniques have been developed to use them in Integrated Pest Management (IPM) programmes against insect pests (Ridgway et al. 1990). In particular, pheromones are widely used in IPM to monitor insect pest populations and to interfere with their behaviour, thus reducing or preventing agricultural damage (Witzgall et al. 2010). On the other hand, signals that operate interspecifically (between different species) are termed 'allelochemicals' and may be called synomones, kairomones or allomones, depending on their ecological and biological functions (Dicke \& Sabelis 1988, Ruther et al. 2002).

The study of the ecological functions of semiochemicals is the main subject of chemical ecology (Ruther et al. 2002, Eisner 2003, Bergstrom 2007, Colazza et al. 2010, WortmanWunder \& Vivanco 2011). In the past two decades, a plethora of studies have demonstrated the importance of chemical cues for ecological processes at the individual, population and ecosystem levels (Takken \& Dicke 2006). However, although there have been considerable advances in understanding, there are still many critical questions that lack answers (Meinwald \& Eisner 2008). The new possibilities offered by genomic and proteomic tools will undoubtedly result in increased understanding over the coming years (Kessler \& Baldwin 2002, Vermeer et al. 2011).

Parasitoids represent fascinating model organisms for evolutionary and ecological studies because of their species richness, ecological impact and economic importance (Godfray 1994, Wajnberg et al. 2008). They belong mainly to two orders, Hymenoptera and Diptera. Within the Hymenoptera, there are about 45 families containing parasitoids. Within the Diptera, most species of parasitoids occur in the family Tachinidae.

Evidence that semiochemicals can modify the behaviour of insect natural enemies has inspired researchers to explore the possibility of using semiochemicals to conserve and/or enhance the efficacy of natural enemies in cropping systems (Pickett et al. 1997, Khan et al. 2008). However, the use of semiochemicals integrated with natural enemies in IPM is still limited, despite the fact that important research has been done in recent years to elucidate the interactions between semiochemicals and natural enemies in a multitrophic context (see Soler, Bezemer \& Harvey, Chapter 4, this volume).

During the last few decades, many studies have investigated the chemically mediated foraging behaviour of parasitoids in an attempt to understand the factors that guide parasitoids to their hosts. In order to provide a quantitative overview of this work, a literature survey was performed. This consisted of interrogating the Scopus database using the term 'parasitoids'. In total, we obtained 10,463 references published within the period 1935-2011 (about 140 papers published per year). Among these, 458 (i.e. $4.38 \%$ ) also used the terms 'synomone', 'kairomone' and/or 'allomone'. Figure 1.1 summarizes the cumulative number of references published over the years and the frequency of the use of the terms 'synomone', 'kairomone' and/or 'allomone'. 


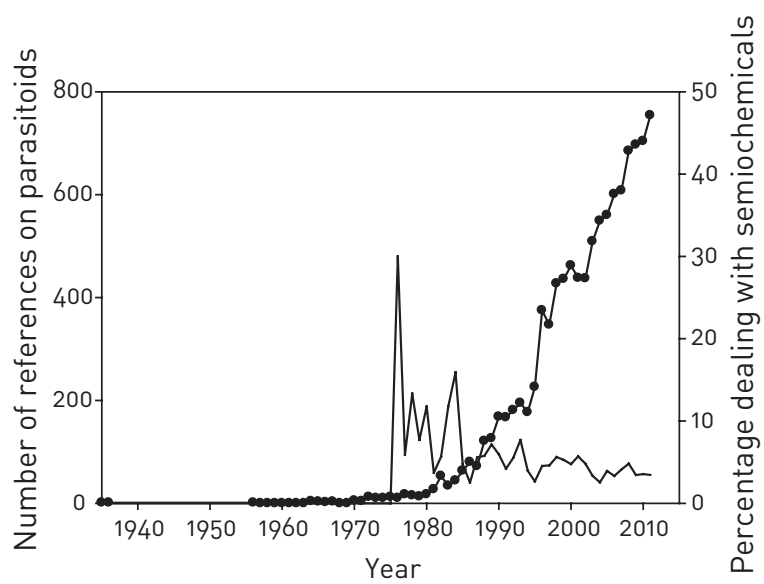

Figure 1.1 Changes in the number of references found in the Scopus abstracts and citations database for the period 1935-2011, using the term 'parasitoids' (line with dots) and the frequency of those that also used the terms 'synomone', 'kairomone' and/or 'allomone' (line without dots).

From about the mid-1970s onwards there has been an increasing interest in publishing papers dealing with parasitoids, coupled with a tendency to focus on chemical ecology. After about a decade, the frequency of papers using the words 'synomone, 'kairomone' and/ or 'allomone' stabilized at around $4.4 \%$. The most likely reason for this is not so much a decline in interest in the chemical ecology of these model animals, but an increase in interest in other aspects of the biology and ecology of parasitoids, for example behavioural ecology (see below). In other words, there was - and still is - a constant interest in understanding the mechanisms involved in the way that insect parasitoids produce and use chemical compounds to communicate with the various different protagonists within their environment (i.e. conspecifics, their hosts, and the plants on which their hosts are living). Unravelling the evolutionary meaning of such a chemical communication network can provide new insights into the ecology of these insects, and especially on how to improve their use for the control of harmful pests in biological control programmes. Therefore, a book covering the current state of knowledge on the chemical ecology of insect parasitoids seems particularly timely and capable of appealing to a large number of potential readers worldwide.

\subsection{Integrating behavioural ecology and chemical ecology in insect parasitoids}

In 2008, Wajnberg et al. (2008) edited a book on the behavioural ecology of insect parasitoids covering cutting-edge research into decision-making processes in insect parasitoids and their implications for biological control. The goal of behavioural ecology is to understand the behavioural decisions adopted by animals to maximize their long-term reproductive success, and for this research theoretical maximization models are frequently used. We visualize this current book on the chemical ecology of insect parasitoids as a 
complementary volume to Wajnberg et al. (2008), extending our understanding and knowledge of insect parasitoids and their use for controlling pests in biological control programmes. After all, chemical ecology - and especially that of insect parasitoids - and behavioural ecology are both based on the accurate observation and analysis of animal behaviour, and the final goal of both volumes is to find ways to improve the efficacy of these insects in controlling pests and protecting crops.

Research efforts in chemical ecology have mainly been based on the development of chemical tools (i) to identify the chemical compounds involved in the way in which insect parasitoids interact with their environment, both at the intra- and interspecific levels; (ii) to understand the associated metabolic pathways; and (iii) to synthesize these compounds for use in enhancing pest control strategies through field-release applications. Such a chemical-based approach has resulted in a rapid improvement in understanding over the past decades, as can be seen in the different chapters within this book. However, we think that it has also precluded the development of theoretical research that aims to understand the optimal decision-making strategy that should be adopted by these insects in terms of releasing and/or perceiving chemical signals in their environment. The fact is that insect parasitoids have been subjected to extreme evolutionary pressure, over the course of time, to use such chemical tools and there are obviously cost/benefit ratio issues that need to be optimized in order to increase the ability of these animals to contribute genetically to the following generations.

The reason that such a theoretical approach has not yet been sufficiently developed, in our view, is most probably because the chemical ecology community mainly consists of scientists who have more expertise in chemistry than in theoretical ecology. There are, however, a handful of studies that have used optimality models to illustrate the chemical ecology of insect parasitoids. For example, Hoffmeister \& Roitberg (1998) developed a theoretical model to identify the optimal persistence duration (or decay rate) of a contact pheromone used by a herbivorous insect to signal the presence of its own eggs to both the marking female and conspecifics. The point is that this pheromone is also exploited by a specialized parasitoid that attacks the herbivore's offspring, so there is an evolutionary game being played between needing to signal the presence of eggs to conspecific females while avoiding their detection by parasitoids. The approach developed by these authors effectively takes into account the physiological costs associated with the marking strategy. Examples like this are still rare, and one goal of the present volume is to foster research in this area, bridging the gap between the behavioural and chemical ecology of insect parasitoids, with the final aim of developing more efficient biological control programmes.

\subsection{The use of chemical ecology to improve the efficacy of insect parasitoids in biological control programmes}

In recent years, significant progress in understanding insect behaviour and advances in analytical chemistry have led to the identification and production of thousands of semiochemical compounds. Important research has been conducted to investigate how these compounds can be used commercially, and many of them are now contributing to established practices in Integrated Pest Management (IPM) (Suckling \& Karg 2000, Witzgall et al. 2010). In this respect, an increasing knowledge of the influence of semiochemicals 
on parasitoid and predator behaviour has opened up new possibilities in pest control strategy.

Semiochemical-based manipulations normally include either 'pheromone-based tactics' or 'allelochemical-based tactics'. Pheromone-based tactics now represent one of the major strategies in ecologically based orchard pest management, leading to considerable success in both direct and indirect insect pest control. The most successful applications for the direct control of pest populations concern the release of sex pheromones to disrupt mating in the target pests (Witzgall et al. 2010). In contrast, allelochemical-based tactics represent a relatively new approach that mainly uses plant volatiles. The most promising application of allelochemical-based tactics involves the use of herbivore-induced plant volatiles (HIPVs) to manipulate the natural enemies of the pest species in order to attract and conserve them in the vicinity of the crops to be protected. HIPVs are semiochemicals that mediate many multitrophic interactions in both above- and below-ground plant-insect communities (Soler et al. 2007, Soler, Bezemer \& Harvey, Chapter 4, this volume). These volatiles have received increased attention for their role in attracting natural enemies of insect pests (Ode, Chapter 2, this volume). In the last decade, the results of several field experiments have been published demonstrating that the release of HIPVs can indeed augment, conserve or enhance the efficacy of natural enemies. However, allelochemical-based tactics, especially based on the use of HIPVs, are lagging far behind the development of applications of pheromones. In this respect, it has to be noted that genetically modified plants have recently been shown to provide new opportunities for semiochemical applications. For example, plants can be engineered to produce $(E)$ - $\beta$-farnesene to mimic the natural aphid alarm response in order to increase foraging by aphid predators and parasitoids (Yu et al. 2012).

\subsection{Overview}

Remarkable advances in our understanding of the chemical ecology of insect parasitoids have occurred in recent years. In this book, we have assembled papers written by internationally recognized experts who are at the forefront of their field. The chapters are organized in order to present the most important knowledge and discoveries made over the past few decades, and on their potential use in pest control strategy. In addition to this introductory chapter, the book contains 12 chapters, organized into two parts. The first part addresses the basic aspects of parasitoid behaviour, and the second focuses on possible strategies for manipulating the behaviour of insect parasitoids to increase their pest control ability by means of chemical cues in different ecosystems and under different agricultural practices. Specific relevant case studies are also presented.

The first part of the book starts with a chapter focusing on plant defence responses to a diversity of pests and abiotic stressors, and their effects on insect parasitoids (Chapter 2). Plants are indeed an important component of the foraging environment of insect parasitoids, orchestrating the presence of complex signalling networks available to these insects. This is the topic addressed by the following chapter, which considers in detail the role of volatile and non-volatile compounds, coupled with biotic and abiotic factors, in shaping the variability of these chemicals in both time and space (Chapter 3 ).

Chemical signals are not only relevant at the above-ground level. There are also signals at the below-ground level that are involved in structuring the ecology of plantinsect interactions. Understanding the role played by these different signals requires a 
multitrophic approach, and that is what is discussed in the following chapter (Chapter 4). Chemical signals are also used by phoretic insect parasitoids that 'hitch-hike' on hosts in order to increase access to potential hosts to attack. Several important and fascinating studies have been carried out on this topic, which is addressed in Chapter 5. In the past decade, astonishing progress has been made to further our understanding of pheromonemediated communication in parasitic wasps, especially for mate finding and recognition, aggregation, or host-marking behaviour, and this is presented in Chapter 6. The two remaining chapters of the first part of the book address the particular case of dipteran tachinid species that demonstrate oviposition strategies which differ from those adopted by hymenopteran wasps (Chapter 7), and the potential consequences of climate change on the chemical ecology of insect parasitoids in general (Chapter 8).

The second part of this book focuses on applications for biological control. The first chapter (Chapter 9) starts by providing a detailed overview of how semiochemicals can be used to manipulate the foraging behaviour of insect parasitoids in order to increase their impact on pest populations. This can be done either through facilitating their ability to locate and attack their hosts, or by increasing their recruitment within agroecosystems. The following chapters then address the application of chemical cues for enhancing the pest control efficacy of parasitic wasps in arable crops (Chapter 10), orchards and vineyards (Chapter 11), organic cropping systems (Chapter 12) and forest trees (Chapter 13).

\subsection{Conclusions}

This book is intended for anyone interested in understanding how insects, and parasitic wasps in particular, use chemical compounds to communicate with others and to discover resources to exploit. The book will be of interest to research scientists and their students working in the academic world (research centres and universities) and also to teachers in graduate schools and universities that teach insect chemical ecology. Furthermore, the background information gathered together in this book could be used to encourage highschool students and stimulate research in the field of chemical ecology of insect parasitoids. Biological control practitioners will also find the technical information needed to improve pest control efficacy through the release of insect parasitoids in the field.

Throughout the book, critical research questions are explicitly identified, acknowledging the gaps in current knowledge. Ultimately, the goal of the book is to foster synergistic research that will eventually lead to a better understanding of the fields of chemical and behavioural ecology of parasitic wasps.

\section{Acknowledgements}

We thank Antonino Cusumano for assistance in interrogating the Scopus database, and Helen Roy and Ezio Peri for critical comments on this chapter. We also wish to thank the referees who read and commented critically on one or more chapters. They include Miguel Borges, Stefano Colazza, Jeff Harvey, Jarmo Holopainen, Martinus Huigens, Yooichi Kainoh, Jocelyn Millar, Satoshi Nakamura, Paul Ode, Tim Paine, Ezio Peri, Guy Poppy, Michael Rostás, Helen Roy, Joachim Ruther, Roxina Soler and Eric Wajnberg. Finally, we express 
our sincere thanks to the staff at Wiley-Blackwell for their excellent help and support during the production of this book.

Although much editing work has been done, the information provided within each chapter remains the sole responsibility of the individual authors.

\section{References}

Bergstrom, G. (2007) Chemical ecology = chemistry + ecology! Pure and Applied Chemistry 79: 2305-23.

Colazza, S., Peri, E., Salerno, G. and Conti, E. (2010) Host searching by egg parasitoids: exploitation of host chemical cues. In: Cônsoli, F.L., Parra, J.R.P. and Zucchi, R.A. (eds) Egg Parasitoids in Agroecosystems with Emphasis on Trichogramma. Springer, Dordrecht, pp. 97-147.

Dawkins, R. and Krebs, J. (1979) Arms races between and within species. Proceedings of the Royal Society of London B 205: 489-511.

Dicke, M. and Sabelis, M.W. (1988) Infochemical terminology: based on cost-benefit analysis rather than origin of compounds. Functional Ecology 2: 131-9.

Eisner, T. (2003) Chemical ecology: can it survive without natural products chemistry? Proceedings of the National Academy of Sciences USA 100: 14517-8.

Godfray, H.C.J. (1994) Parasitoids: Behavioural and Evolutionary Ecology. Princeton University Press, Princeton, NJ.

Hoffmeister, T.S. and Roitberg, B.D. (1998) Evolution of signal persistence under predator exploitation. Ecoscience 5: 312-20.

Kessler, A. and Baldwin, I.T. (2002) Plant responses to insect herbivory: The emerging molecular analysis. Annual Review of Plant Biology 53: 299-328.

Khan, Z.R., James, D.G., Midega, C.A.O. and Pickett, C.H. (2008) Chemical ecology and conservation biological control. Biological Control 45: 210-24.

Krebs, J.R. and Davies, N.B. (1987) An Introduction to Behavioural Ecology. Blackwell Publishing Ltd., Oxford.

Meinwald, J. and Eisner, T. (2008) Chemical ecology in retrospect and prospect. Proceedings of the National Academy of Sciences USA 105: 4539-40.

Nordlund, D.A. (1981) Semiochemicals: a review of the terminology. In: Nordlund, D.A., Jones, R.J. and Lewis, W.J. (eds) Semiochemicals: Their Role in Pest Control. John Wiley \& Sons, New York, pp. 13-28.

Pickett, J.A., Wadhams, L.J. and Woodcock, C.M. (1997) Developing sustainable pest control from chemical ecology. Agriculture, Ecosystems and Environment 64: 149-56.

Ridgway, R.L., Silverstein, R.M. and Inscoe, M.N. (1990) Behavior-Modifying Chemicals for Insect Management: Applications of Pheromones and other Attractants. Marcel Dekker, New York.

Ruther, J., Meiners, T. and Steidle, J.L.M. (2002) Rich in phenomena - lacking in terms: a classification of kairomones. Chemoecology 12: 161-7.

Soler, R., Harvey, J.A. and Bezemer, T.M. (2007) Foraging efficiency of a parasitoid of a leaf herbivore is influenced by root herbivory on neighbouring plants. Functional Ecology 21: 969-74.

Suckling, D.M. and Karg, G. (2000) Pheromones and semiochemicals. In: Rechcigl, J. and Rechcigl, N. (eds) Biological and Biotechnical Control of Insect Pests. CRC Press, Boca Raton, FL, pp. 63-99.

Takken, W. and Dicke, M. (2006) Chemical ecology: a multidisciplinary approach. In: Dicke, M. and Takken, W. (eds) PhD Spring School Chemical Communication: From Gene to Ecosystem. Wageningen, 19-23 March 2005. Springer, Dordrecht, pp. 1-8.

Vermeer, K.M.C.A., Dicke, M. and de Jong, P.W. (2011) The potential of a population genomics approach to analyse geographic mosaics of plant-insect coevolution. Evolutionary Ecology 25: 977-92. 
Vet, L.E.M. and Dicke, M. (1992) Ecology of infochemical use by natural enemies in a tritrophic context. Annual Review of Entomology 37: 141-72.

Vinson, S.B. (1985) The behavior of parasitoids. In: Kerkut, G.A. and Gilbert, L.I. (eds) Comprehensive Insect Physiology, Biochemistry, and Pharmacology, Vol. 9. Pergamon Press, Elmsford, NY, pp. 417-69.

Wajnberg, E., Bernstein, C. and van Alphen, J. (2008) Behavioral Ecology of Insect Parasitoids: From Theoretical Approaches to Field Applications. Blackwell Publishing Ltd., Oxford.

Witzgall, P., Kirsch, P. and Cork, A. (2010) Sex pheromones and their impact on pest management. Journal of Chemical Ecology 36: 80-100.

Wortman-Wunder, E. and Vivanco, J.M. (2011) Chemical ecology: definition and famous examples. In: Vivanco, J.M. and Weir, T. (eds) Chemical Biology of the Tropics: An Interdisciplinary Approach. Springer-Verlag, Berlin, Heidelberg, pp. 15-26.

Wyatt, T.D. (2010) Pheromones and signature mixtures: defining species-wide signals and variable cues for individuality in both invertebrates and vertebrates. Journal of Comparative Physiology A 196: 685-700.

Yu, X.D., Pickett, J., Ma, Y.Z., Bruce, T., Napier, J., Jones, H.D. and Xia, L.Q. (2012) Metabolic engineering of plant-derived $(E)$ - $\beta$-farnesene synthase genes for a novel type of aphid-resistant genetically modified crop plants. Journal of Integrative Plant Biology 54: 282-99. 